\title{
The Statistical Properties of Spin-One DKP Oscillator under an External Magnetic Field in Noncommutative Space
}

\author{
S. Hassanabadi and M. Ghominejad \\ Physics Department, Semnan University, Semnan, Iran \\ Correspondence should be addressed to M. Ghominejad; mghominejad@semnan.ac.ir \\ Received 30 January 2014; Revised 27 March 2014; Accepted 14 April 2014; Published 22 May 2014 \\ Academic Editor: Chao-Qiang Geng
}

Copyright (c) 2014 S. Hassanabadi and M. Ghominejad. This is an open access article distributed under the Creative Commons Attribution License, which permits unrestricted use, distribution, and reproduction in any medium, provided the original work is properly cited. The publication of this article was funded by SCOAP ${ }^{3}$.

\begin{abstract}
The spin-one Duffin-Kemmer-Petiau oscillator in uniform magnetic field is studied in noncommutative formalism. The corresponding energy is obtained and thereby the corresponding thermal properties are obtained for both commutative and noncommutative cases.
\end{abstract}

\section{Introduction}

The first-order relativistic Duffin-Kemmer-Petiau (DKP) equation has been frequently used to study interactions of spin-one/zero particles [1] in various studies of particle physics. As typical examples, DKP equation has been also used to study deuteron-nucleus scattering [2], $\alpha$-nucleus elastic scattering, meson-nuclear interaction [3,4], and the scattering of $K+$ nucleus in the presence of an AharonovBohm potential [5].

On the other hand, we have motivating evidences that we have to work on the noncommutative (NC) formulation of quantum mechanics where the position or momentum operators have nonvanishing commutations. During the past years, some important problems have been investigated in the NC formalism [6-11]. Typical examples of such cases include an open string attached to $D$-branes in the presence of background B-field inducing noncommutativity in its both end points [12-15], the quantum Hall effect [16] and the DKP oscillator [17]. The interface with solid-state physics and semiconductor theories are also studied in [17-20].

Due to the physical significance of the magnetic field, Pacheco et al. studied the thermal properties of the onedimensional Dirac oscillator problem [21, 22]. Although there are very recent papers which consider the NC DKP oscillator, no one, to our best knowledge, has discussed the corresponding thermodynamical properties. In our work, we investigate the spin-one DKP oscillator with the magnetic field in NC phase-space and search for the corresponding thermodynamical properties including partition function $Z$, mean internal energy $U$, Helmholtz free energy $F$, specific heat capacity $C_{v}$, and entropy $S$.

\section{Noncommutative Quantum Mechanics}

One way to deal with the NC space is to construct a new kind of field theory, changing the standard product of the fields by the star product (Weyl-Moyal)

$$
(f * g)(x)=\exp \left(i \theta^{i j} \partial_{x^{i}} \partial_{x^{j}}\right) f\left(x^{i}\right) g\left(x^{j}\right),
$$

where $\theta^{i j}=\theta \varepsilon^{i j}$ is an antisymmetric matrix with real elements and represents the noncommutativity of the space. In (1), $f$ and $g$ are both infinitely differentiable functions [23] and the $\mathrm{NC}$ space corresponds to the commutation relations

$$
\left[\hat{x}^{i}, \widehat{x}^{j}\right]=i \theta^{i j}, \quad\left[\hat{p}^{i}, \hat{p}^{j}\right]=0, \quad\left[\hat{x}^{i}, \hat{p}^{j}\right]=i \hbar \delta^{i j} .
$$

To map the ordinary quantum mechanics into its NC version, it is sufficient to imply the so-called Bopp-shift. The latter implies the transformation

$$
\hat{x}^{i} \longrightarrow x^{i}-\frac{1}{2} \theta \varepsilon^{i j} p^{j}
$$


As we know, the DKP equation for free particles with spinone and spin-zero is (in natural units $\hbar=c=1$ )

$$
\left(i \beta^{\mu} \partial_{\mu}-m\right) \psi=0
$$

where the DKP matrices satisfy the algebra

$$
\begin{gathered}
\beta^{\mu} \beta^{\nu} \beta^{\lambda}+\beta^{\lambda} \beta^{\nu} \beta^{\mu}=g^{\mu \nu} \beta^{\lambda}+g^{\lambda \nu} \beta^{\mu}, \\
g^{\mu \nu}=\operatorname{diag}(1,-1,-1,-1), \quad \mu=0,1,2,3 .
\end{gathered}
$$

Within the ten-dimensional representation of spin-one DKP equation,

$$
\begin{aligned}
& \beta^{0}=\left|\begin{array}{llll}
0_{3 \times 3} & \overline{0}_{1 \times 3} & \overline{0}_{1 \times 3} & \overline{0}_{1 \times 3} \\
\overline{0}_{1 \times 3}^{T} & 0_{3 \times 3} & I_{3 \times 3} & 0_{3 \times 3} \\
\overline{0}_{1 \times 3}^{T} & I_{3 \times 3} & 0_{3 \times 3} & 0_{3 \times 3} \\
\overline{0}_{1 \times 3}^{T} & 0_{3 \times 3} & 0_{3 \times 3} & 0_{3 \times 3}
\end{array}\right|, \\
& \beta^{i}=\left|\begin{array}{llll}
0_{3 \times 3} & \overline{0}_{1 \times 3} & e_{i} & \overline{0}_{1 \times 3} \\
\overline{0}_{1 \times 3}^{T} & 0_{3 \times 3} & 0_{3 \times 3} & -i S_{i} \\
-e_{i}^{T} & 0_{3 \times 3} & 0_{3 \times 3} & 0_{3 \times 3} \\
\overline{0}_{1 \times 3}^{T} & -i S_{i} & 0_{3 \times 3} & 0_{3 \times 3}
\end{array}\right|,
\end{aligned}
$$

with the $S_{i}$ matrices being $3 \times 3$ ones, $\left(S_{i}\right)_{j k}=-i \varepsilon_{i j k}$, where $\varepsilon_{i j k}$ is the Levi-Civita tensor, $e_{i}$ matrices are $1 \times 3$ ones, and $\left(e_{i}\right)_{1 j}=\delta_{i j}$, that is, $e_{1}=(1,0,0), e_{2}=(0,1,0), e_{3}=$ $(0,0,1) . I$ and $0_{3 \times 3}$, respectively, represent unit and null $3 \times$ 3 matrices and $\overline{0}$ are $1 \times 3$ ones [24-27]. By considering a magnetic field of the form $\vec{B}=(0,0, B)=B \widehat{k}$ in the symmetric gauge $\vec{A}(\vec{r})=\left(-x_{2} B / 2, x_{1} B / 2,0\right)=(\vec{B} \times \vec{r}) / 2$, the momentum is transformed as $\vec{p} \rightarrow(\vec{p}-e \vec{A} / c)$. The DKP equation in $(2+1)$-dimensions under a uniform magnetic field has the form $(e=1)$

$$
\begin{gathered}
\left\{\beta^{0} E_{n, m}-\beta^{1}\left(\widehat{p}_{1}+\frac{B}{2} \widehat{x}_{2}-i m w \eta^{0} \widehat{x}_{1}\right)\right. \\
\left.-\beta^{2}\left(\widehat{p}_{2}-\frac{B}{2} \widehat{x}_{1}-i m w \eta^{0} \widehat{x}_{1}\right)-m\right\} \psi_{n, l}(x, y, t)=0, \\
\psi_{n, l}(x, y, t)=e^{-i E_{n, l} t} \varphi_{n, l}(x, y),
\end{gathered}
$$

where

$$
\varphi_{n, l}(x, y)=\left(\varphi_{n, l}^{1}, \varphi_{n, l}^{2}, \varphi_{n, l}^{3}, \varphi_{n, l}^{4}, \varphi_{n, l}^{5}, \varphi_{n, l}^{6}, \varphi_{n, l}^{7}, \varphi_{n, l}^{8}, \varphi_{n, l}^{9}, \varphi_{n, l}^{10}\right)^{T}
$$

Combining the above equations, we have the system of equations

$$
\begin{aligned}
& \left(\widehat{p}_{1}+\frac{B}{2} \widehat{x}_{2}-i m w \widehat{x}_{1}\right) \varphi_{n, m}^{5}(x, y) \\
& +\left(\widehat{p}_{2}-\frac{B}{2} \widehat{x}_{1}-i m w \widehat{x}_{2}\right) \varphi_{n, m}^{6}(x, y)-m \varphi_{n, m}^{1}(x, y)=0 \\
& E_{n, m} \varphi_{n, m}^{5}(x, y)+\left(\widehat{p}_{2}-\frac{B}{2} \widehat{x}_{1}+i m w \widehat{x}_{2}\right) \varphi_{n, m}^{10}(x, y) \\
& \quad-m \varphi_{n, m}^{2}(x, y)=0, \\
& E_{n, m} \varphi_{n, m}^{6}(x, y)-\left(\widehat{p}_{1}+\frac{B}{2} \widehat{x}_{2}+i m w \widehat{x}_{1}\right) \varphi_{n, m}^{10}(x, y) \\
& \quad-m \varphi_{n, m}^{3}(x, y)=0, \\
& -\left(\widehat{p}_{n, m} \varphi_{n, m}^{7}(x, y)-\left(\widehat{p}_{1}+\frac{B}{2} \widehat{x}_{2}+i m w \widehat{x}_{1}\right) \varphi_{n, m}^{9}(x, y)\right. \\
& +\left(\widehat{p}_{2}-\frac{B}{2} \widehat{x}_{1}+i m w \widehat{x}_{2}\right) \varphi_{n, m}^{8}(x, y)-m \varphi_{n, m}^{4}(x, y)=0 \\
& -\left(\widehat{p}_{1}-\frac{B}{2} \widehat{x}_{2}-i m w \widehat{x}_{1}\right) \varphi_{n, m}^{4}(x, y)-m \varphi_{n, m}^{9}(x, y)=0 \\
& \quad-m \widehat{x}_{n, m}^{6}(x, y)=0, \\
& \quad-m \varphi_{n, m}^{5}(x, y)=0, \\
& E_{n, m} \varphi_{n, m}^{2}(x, y)-\left(\widehat{p}_{1}+\frac{B}{2} \widehat{x}_{2}+i m w \widehat{x}_{1}\right) \varphi_{n, m}^{1}(x, y)
\end{aligned}
$$

Assuming $\varphi_{n, m}^{2}(x, y)=\varphi_{n, m}^{3}(x, y)=0$, from (9a)-(9k) we have

$$
\begin{aligned}
& E_{n, m} \varphi_{n, m}^{7}(x, y)-\left(\widehat{p}_{1}+\frac{B}{2} \widehat{x}_{2}+i m w \widehat{x}_{1}\right) \varphi_{n, m}^{9}(x, y) \\
& +\left(\widehat{p}_{2}-\frac{B}{2} \widehat{x}_{1}+i m w \widehat{x}_{2}\right) \varphi_{n, m}^{8}(x, y)-m \varphi_{n, m}^{4}(x, y)=0,
\end{aligned}
$$




$$
\begin{gathered}
E_{n, m} \varphi_{n, m}^{4}(x, y)-m \varphi_{n, m}^{7}(x, y)=0 \\
-\left(\widehat{p}_{2}-\frac{B}{2} \widehat{x}_{1}-i m w \widehat{x}_{2}\right) \varphi_{n, m}^{4}(x, y)-m \varphi_{n, m}^{8}(x, y)=0 \\
-\left(\widehat{p}_{1}+\frac{B}{2} \widehat{x}_{2}-i m w \widehat{x}_{1}\right) \varphi_{n, m}^{4}(x, y)-m \varphi_{n, m}^{9}(x, y)=0
\end{gathered}
$$

Equations (10a)-(10d), after some algebra, give

$$
\begin{aligned}
& \left\{E_{n, l}^{2}-\left(\widehat{p}_{1}+\frac{B}{2} \widehat{x}_{2}+i m w \widehat{x}_{1}\right)\left(\widehat{p}_{1}+\frac{B}{2} \widehat{x}_{2}-i m w \widehat{x}_{1}\right)\right. \\
& \left.-\left(\widehat{p}_{2}-\frac{B}{2} \widehat{x}_{1}+i m w \widehat{x}_{2}\right)\left(\widehat{p}_{2}-\frac{B}{2} \widehat{x}_{1}-i m w \widehat{x}_{2}\right)-m^{2}\right\} \\
& \quad \times \varphi_{n, l}^{4}(x, y)=0 .
\end{aligned}
$$

To map the problem into the NC formulation, we consider

$$
\widehat{x}_{1}=x_{1}-\frac{\theta P_{2}}{2}, \quad \widehat{x}_{2}=x_{2}+\frac{\theta P_{1}}{2},
$$

which transform (11) into

$$
\begin{aligned}
& \left\{E_{n, l}^{2}-p^{2}\left(1+\frac{m^{2} w^{2} \theta^{2}}{4}+\frac{B \theta}{2}+\frac{B^{2} \theta^{2}}{16}\right)\right. \\
& -r^{2}\left(m^{2} w^{2}+\frac{B^{2}}{4}\right)+\left(\frac{B^{2} \theta}{4}+m^{2} w^{2} \theta+B\right) L_{z}+m w B \theta \\
& \left.+2 m w-m^{2}\right\} \varphi_{n, l}^{4}(x, y)=0 .
\end{aligned}
$$

Proposing the well-known separation of variables $\varphi_{n, l}^{4}(r, \phi)=$ $R_{n}^{4}(r) e^{i l \phi}$ followed by a transformation of the form $R(r)=$ $r^{-1 / 2} u(r)$, (13) gives

$$
\left\{d_{1} \frac{d^{2}}{d r^{2}}+\frac{1}{r^{2}}\left(-l^{2}+\frac{1}{4}\right) d_{1}+d_{2}-r^{2} d_{3}\right\} u(r)=0,
$$

where

$$
\begin{gathered}
d_{1}=1+\frac{B \theta}{2}+\frac{B^{2} \theta^{2}}{16}+\frac{m^{2} w^{2} \theta^{2}}{4} \\
d_{2}=E_{n, l}^{2}-m^{2}+l\left(B+\frac{B^{2} \theta}{4}+m^{2} w^{2} \theta-m w-2 m w\right) \\
d_{3}=\frac{B^{2}}{4}+m^{2} w^{2}
\end{gathered}
$$

Proposing a change of variable of the form $s=r^{2}$, (14) transforms into

$$
\left\{\frac{d^{2}}{d s^{2}}+\frac{1}{2 s} \frac{d}{d s}+\frac{1}{s^{2}}\left\{d_{4}+d_{5} s+d_{6} s^{2}\right\}\right\} u(s)=0,
$$

where

$$
d_{4}=\frac{\left(1 / 4-l^{2}\right)}{4}, \quad d_{5}=\frac{d_{2}}{4 d_{1}}, \quad d_{6}=-\frac{d_{3}}{4 d_{1}} .
$$

To obtain the wave function, we simply use the parametric Nikiforov-Uvarov (NU) method from the Appendix. A simple comparison reveals the correspondence

$$
\begin{gathered}
\alpha_{1}=\frac{1}{2}, \quad \alpha_{2}=\alpha_{3}=0, \quad \xi_{1}=-d_{6}, \\
\xi_{2}=d_{5}, \quad \xi_{3}=-d_{4}, \\
\alpha_{4}=\frac{1}{4}, \quad \alpha_{5}=0, \quad \alpha_{6}=-d_{6}, \quad \alpha_{7}=-d_{5}, \\
\alpha_{8}=\frac{1}{16}-d_{4}, \quad \alpha_{9}=-d_{6} .
\end{gathered}
$$

Using (20), the energy relation is found as

$$
E_{n, l}= \pm m \sqrt{\frac{4 \sqrt{d_{3} d_{1}} n+2 \sqrt{d_{3} d_{1}}-k+\sqrt{d_{3} d_{1}\left(+1-16 d_{4}\right)}}{m^{2}}}
$$

or

$$
E_{n, l}^{ \pm}= \pm m \sqrt{\eta+4 \bar{\lambda}} n, \quad n=1,2, \ldots, l=0,1, \ldots,
$$

with

$$
\begin{gathered}
\bar{\lambda}=\frac{\sqrt{d_{3} d_{1}}}{m^{2}}, \\
\eta=\frac{2 \sqrt{d_{3} d_{1}}-k+\sqrt{d_{3} d_{1}\left(1-16 d_{4}\right)}}{m^{2}} .
\end{gathered}
$$

The partition function of the DKP oscillator at temperature $T$ is obtained from

$$
Z(V, T)=\sum_{n=0}^{n} e^{-\beta\left(E_{n}-E_{0}\right)},
$$

where $\beta=1 / k_{B} T$ and $k_{B}$ is the Boltzmann constant. All thermodynamical quantities of a physical system are obtained through the partition function [28]. We start with the Helmholtz free energy $F$ which is defined as

$$
F(N, V, T)=-k_{B} T \ln Z(V, T) .
$$

Once the Helmholtz free energy is obtained, other statistical quantities are obtained in a straightforward manner. The mean energy $U$ and the specific heat capacity at constant volume are respectively given by

$$
\begin{gathered}
U=-\frac{\partial \ln Z}{\partial \beta}=-N \frac{\partial \ln Z_{1}}{\partial \beta}=N m c^{2} \tau^{2} \frac{\partial \ln Z_{1}}{\partial \tau}, \\
C_{v}=\frac{\partial\langle U\rangle}{\partial T}=\frac{k_{B}}{m c^{2}} \frac{\partial\langle U\rangle}{\partial \tau}=-k_{B} \beta^{2} \frac{\partial\langle U\rangle}{\partial \beta} .
\end{gathered}
$$




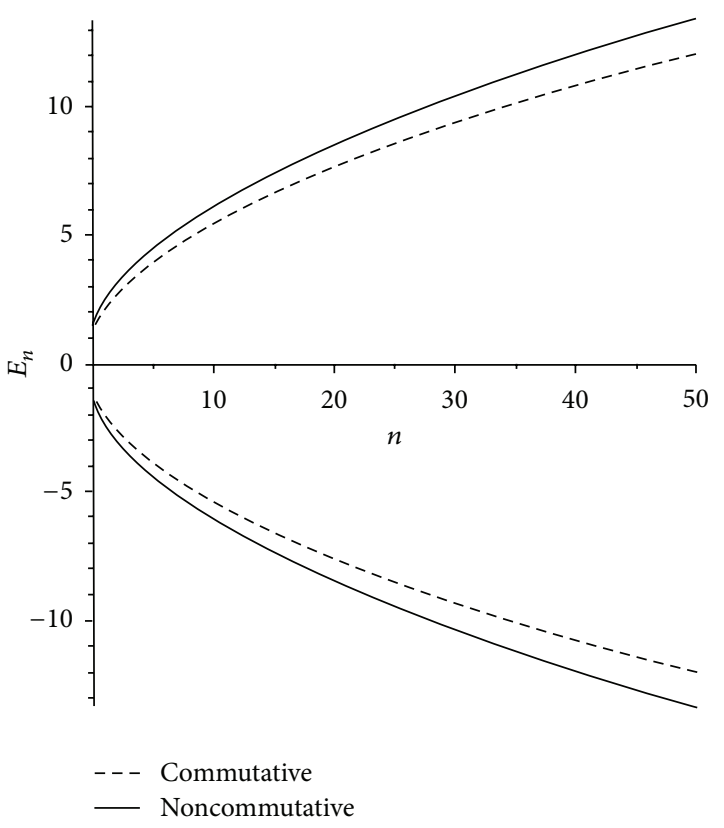

FIGURE 1: The comparison of the $E_{n}$ for both commutative and noncommutative space.

Entropy is related to the other quantities with the relations

$$
\begin{aligned}
S & =-N \frac{\partial}{\partial T} F=N k_{B} \ln Z_{1}+N k_{B} T \frac{\partial}{\partial T}\left(\ln Z_{1}\right) \\
& =N k_{B} \ln Z_{1}-N k_{B} \beta \frac{\partial}{\partial \beta}\left(\ln Z_{1}\right) .
\end{aligned}
$$

Accordingly, the NC DKP oscillator partition function is

$$
Z_{1}=\sum_{n=0}^{n} e^{-\beta m c^{2} \sqrt{\eta+4 \bar{\lambda} n}}=e^{\beta m c^{2} \sqrt{\eta}} \sum_{n=0}^{n} e^{-\beta m c^{2} \sqrt{\eta+4 \bar{\lambda} n}} .
$$

By using the Euler-Maclaurin integration formula [29] (see Appendix B) and after a simple calculation, we calculate the partition function as

$$
\begin{aligned}
Z_{1}= & \left(\frac{1}{2}+\frac{\sqrt{\eta}}{2 \bar{\lambda}} \tau+\frac{1}{2 \bar{\lambda}} \tau^{2}+\left(\frac{\bar{\lambda}}{6 \sqrt{\eta}}-\frac{\bar{\lambda}^{3}}{30 \sqrt{\eta^{5}}}\right) \frac{1}{\tau}\right. \\
& \left.-\frac{1}{30 \eta^{2}} \frac{\bar{\lambda}^{3}}{\tau^{2}}-\frac{\bar{\lambda}^{3}}{60 \sqrt{\eta^{3}} \tau^{3}}\right),
\end{aligned}
$$

where $\tau=1 / \beta m c^{2}$. We can extend DKP oscillator partition function to an $N$-body system (without any interaction between the ingredients) via $Z=\left[Z_{1}\right]^{N}$.

\section{Conclusion}

We obtained the statistical quantities of the charged DKP oscillator in a uniform magnetic field in the NC space in
TABLE 1: Energy spectra $E_{n l}$ for various quantum numbers $n, l$ for $\hbar=c=1, m=e=1, \omega=0.5, \theta=0.8$, and $B=1$ in noncommutative case.

\begin{tabular}{ll}
\hline$|n, l\rangle$ & $E_{n, l}^{(\mathrm{NCS})}$ \\
\hline$|1,0\rangle$ & 2.1820 \\
$|1,1\rangle$ & 2.2542 \\
$|1,2\rangle$ & 2.3242 \\
$|1,3\rangle$ & 2.3922 \\
$|1,4\rangle$ & 2.4583 \\
$|1,5\rangle$ & 2.5226 \\
$|2,0\rangle$ & 2.8639 \\
$|2,1\rangle$ & 2.9193 \\
$|2,2\rangle$ & 2.9737 \\
$|2,3\rangle$ & 3.0271 \\
$|2,4\rangle$ & 3.0796 \\
$|2,5\rangle$ & 3.1312 \\
$|3,0\rangle$ & 3.4122 \\
$|3,1\rangle$ & 3.4588 \\
$|3,2\rangle$ & 3.5048 \\
$|3,3\rangle$ & 3.5503 \\
$|3,4\rangle$ & 3.5951 \\
$|3,5\rangle$ & 3.6394 \\
$|4,0\rangle$ & 3.8838 \\
$|4,1\rangle$ & 3.9248 \\
$|4,2\rangle$ & 3.9654 \\
$|4,3\rangle$ & 4.0056 \\
\hline
\end{tabular}

$(2+1)$-dimensions in an exact analytical manner and thereby the effect of the noncommutative parameter on the thermal properties was obtained. Tables 1 and 2 give the energies in both commutative and NC cases. In Figure 1, we have depicted the energy $\left(E_{n}\right)$ of the relativistic spin-one bosons as a function of $n$. We see that the effect of the NC parameter on the energy levels is considerable. The thermodynamic quantities in the NC and commutative cases are, respectively, plotted in Figures 2 and 3. In Figure 2, the behavior of the partition function versus $T$ is plotted for various values of the magnetic field. It shows that with increasing $T$ the partition function has an increasing behavior. In Figure 3, where the Helmholtz free energy is plotted versus $T$, it is seen that the energy decreases in a nearly linear behavior for increasing $T$.

\section{Appendices}

\section{A. The Parametric NU Method}

The NU method, named after Nikiforov and Uvarov, can solve a wide class of ordinary differential equations at most of second order. It has been already applied to other wave equations of quantum mechanics including Schrödinger, Dirac, Klein-Gordon, and Duffin-Kemmer-Petiau (DKP) equations. Here, for the sake of simplicity, we use its parametric version 
TABLE 2: Energy spectra $E_{n, l}$ for various quantum numbers $n, l$ for $\hbar=c=1, m=e=1, \omega=0.5, \theta=0$, and $B=1$ for commutative space.

\begin{tabular}{lc}
\hline$|n, l\rangle$ & $E_{n, l}^{(\mathrm{CS})}$ \\
\hline$|1,0\rangle$ & 2.0597 \\
$|1,1\rangle$ & 2.1579 \\
$|1,2\rangle$ & 2.2519 \\
$|1,3\rangle$ & 2.3402 \\
$|1,4\rangle$ & 2.4288 \\
$|1,5\rangle$ & 2.5127 \\
$|2,0\rangle$ & 2.6591 \\
$|2,1\rangle$ & 2.7359 \\
$|2,2\rangle$ & 2.8106 \\
$|2,3\rangle$ & 3.8833 \\
$|2,4\rangle$ & 3.9543 \\
$|2,5\rangle$ & 3.0235 \\
$|3,0\rangle$ & 3.1463 \\
$|3,1\rangle$ & 3.2114 \\
$|3,2\rangle$ & 3.2753 \\
$|3,3\rangle$ & 3.3379 \\
$|3,4\rangle$ & 3.3994 \\
$|3,5\rangle$ & 3.4598 \\
$|4,0\rangle$ & 3.5676 \\
$|4,1\rangle$ & 3.6252 \\
$|4,2\rangle$ & 3.6818 \\
$|4,3\rangle$ & 3.7377 \\
\hline
\end{tabular}

which solves a second-order differential equation of the form $[30,31]$,

$$
\left[\frac{d^{2}}{d s^{2}}+\frac{\alpha_{1}-\alpha_{2} s}{s\left(1-\alpha_{3} s\right)} \frac{d}{d s}+\frac{-\xi_{1} s^{2}+\xi_{2} s-\xi_{3}}{\left[s\left(1-\alpha_{3} s\right)\right]^{2}}\right] \psi(s)=0 .
$$

In the NU method, the energy eigenvalues satisfy $[30,31]$

$$
\begin{aligned}
& \alpha_{2} n-(2 n+1) \alpha_{5}+(2 n+1)\left(\sqrt{\alpha_{9}}+\alpha_{3} \sqrt{\alpha_{8}}\right) \\
& \quad+n(n-1) \alpha_{3}+\alpha_{7}+2 \alpha_{3} \alpha_{8}+2 \sqrt{\alpha_{8} \alpha_{9}}=0,
\end{aligned}
$$

where

$$
\begin{gathered}
\alpha_{4}=\frac{1}{2}\left(1-\alpha_{1}\right), \quad \alpha_{5}=\frac{1}{2}\left(\alpha_{2}-2 \alpha_{3}\right), \\
\alpha_{6}=\alpha_{5}^{2}+\xi_{1}, \quad \alpha_{7}=2 \alpha_{4} \alpha_{5}-\xi_{2}, \\
\alpha_{8}=\alpha_{4}^{2}+\xi_{3}, \quad \alpha_{9}=\alpha_{3} \alpha_{7}+\alpha_{3}^{2} \alpha_{8}+\alpha_{6} .
\end{gathered}
$$

\section{B. Formulation of Euler-Maclaurin}

The partition function $Z$ of the DKP oscillator is

$$
Z(V, T)=\sum_{n=0}^{n} e^{-\beta\left(E_{n}-E_{0}\right)}=e^{\beta \sqrt{b}} \sum_{n=0}^{\infty} e^{-\beta \sqrt{a x+b}} .
$$

We should test the convergence of the series of (B.1). For that, we apply the integral test which shows that the series and the

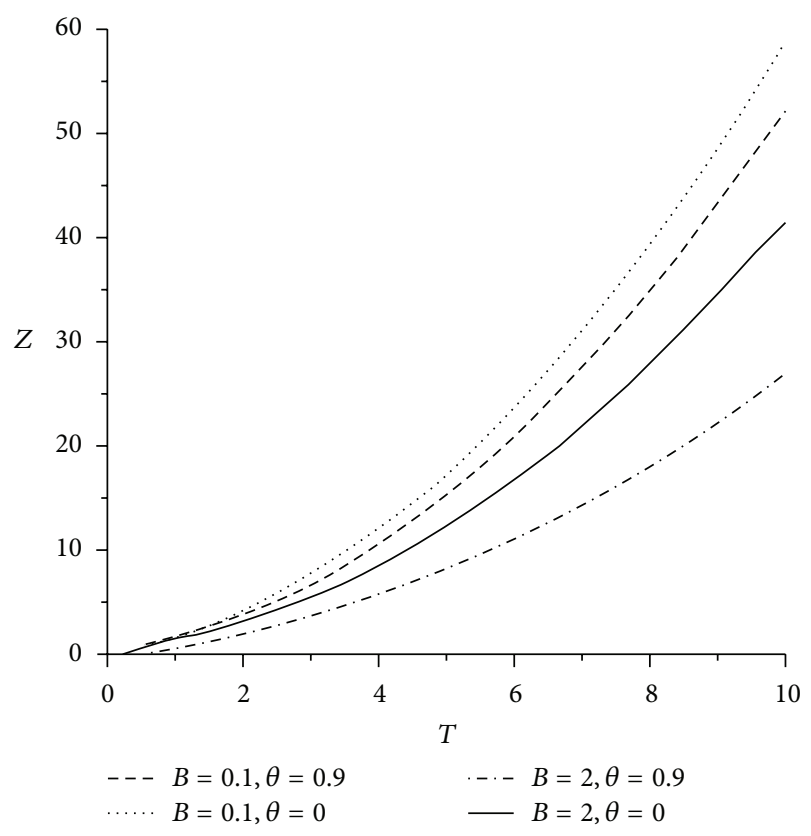

FIGURE 2: The comparison of the partition functions for both commutative and noncommutative cases.

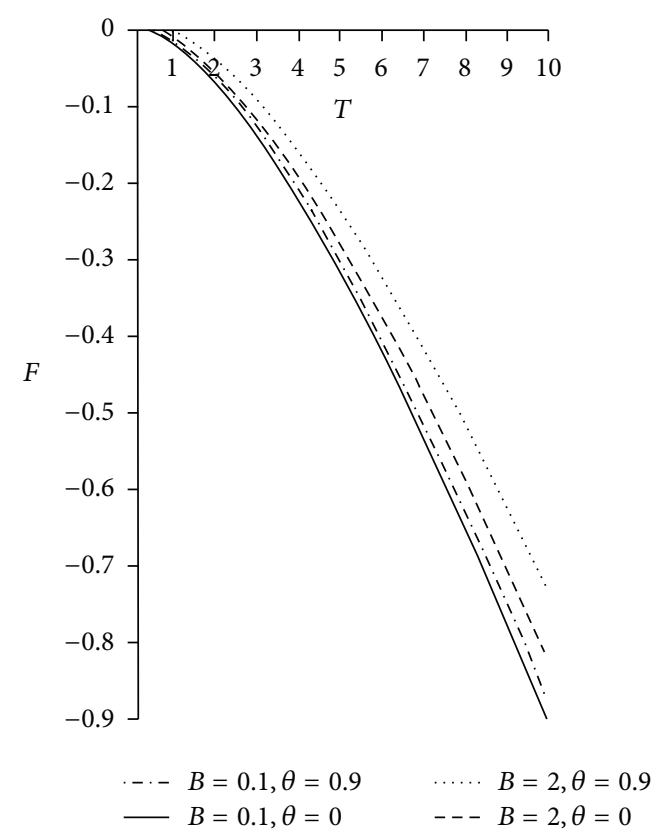

Figure 3: The comparison of the Helmholtz free energy $F$ for both commutative and noncommutative cases.

integral converge or diverge together. So, from (B.1), we can see that the function $f(x)$, where

$$
f(x)=e^{-\beta \sqrt{a x+b}},
$$

is a decreasing positive function, and the integral

$$
\int_{0}^{\infty} f(x) d x=\frac{2}{a \beta} e^{-\beta \sqrt{b}}(1+\beta \sqrt{b}),
$$


is convergent. In order to evaluate this function, we use the Euler-Maclaurin formula defined as follows:

$$
\sum_{n=0}^{n} f(x)=\frac{1}{2} f(0)+\int_{0}^{\infty} f(x) d x-\sum_{p=1}^{\infty} \frac{1}{(2 p) !} B_{2 p} f^{(2 p-1)}(0) .
$$

Here, $B_{2 p}$ are the Bernoulli numbers, $B_{2}=1 / 6, B_{4}=$ $-1 / 30, \ldots$. In our case, we have used

$$
f^{(1)}(x)=\frac{a \beta}{2 \sqrt{b+a x}} e^{-\beta \sqrt{b+a x}}
$$

$$
\begin{aligned}
& f^{(3)}(x) \\
& \quad=\left[\frac{-3 \beta a^{3}}{8(b+a x)^{5 / 2}}-\frac{3 \beta^{2} a^{3}}{8(b+a x)^{2}}-\frac{\beta^{3} a^{3}}{8(b+a x)^{3 / 2}}\right] e^{-\beta \sqrt{b+a x}} .
\end{aligned}
$$

Using above equations (B.4), (B.5), and (B.6), the partition function can be then written as

$$
z=\frac{1}{2}+\frac{2}{a_{1} \beta^{2}}\left(1+\beta \sqrt{b_{1}}\right)-\frac{B_{2}}{2} f^{(1)}-\frac{B_{4}}{24} f^{(3)}+\cdots
$$

\section{Conflict of Interests}

The authors declare that there is no conflict of interests regarding the publication of this paper.

\section{Acknowledgment}

We wish to give our sincere gratitude to the referee for his technical comments.

\section{References}

[1] Y. Nedjadi and R. C. Barrett, "On the properties of the DuffinKemmer-Petiau equation," Journal of Physics G: Nuclear and Particle Physics, vol. 19, no. 1, pp. 87-98, 1993.

[2] R. E. Kozack, B. C. Clark, S. Hama, V. K. Mishra, R. L. Mercer, and L. Ray, Physical Review C, vol. 40, 1989.

[3] V. N. Gribov, "QCD at large and short distances (annotated version)," European Physical Journal C, vol. 10, no. 1, pp. 71-90, 1999.

[4] L. K. Keer, B. C. Clark, S. Hama, L. Ray, and G. W. Hoffmann, "Theoretical and experimental $\mathrm{K}++$ nucleus total and reaction cross sections from the KDP-RIA model," Progress of Theoretical Physics, vol. 103, no. 2, pp. 321-335, 2000.

[5] A. Boumali, "Particule de spin-1 dans un potentiel d'AharonovBohm," Canadian Journal of Physics, vol. 85, no. 12, pp. 14171429, 2007.

[6] G.-F. Wei, C.-Y. Long, Z.-W. Long, and S.-J. Qin, Chinese Physics C, vol. 32, no. 4, 2008.

[7] H. Hassanabadi, Z. Molaee, and S. Zarrinkamar, "DKP oscillator in the presence of magnetic field in $(1+2)$-dimensions for spin-zero and spin-one particles in noncommutative phase space," The European Physical Journal, vol. 72, p. 2217, 2012.
[8] K. Li, J. H. Wang, and C. Y. Chen, Modern Physics Letters A: Articles and Fields, Gravitation, Cosmology, Nuclear Physics, vol. 20, no. 34, 2005.

[9] A. Connes, M. R. Douglas, and A. Schwarz, "Noncommutative geometry and Matrix theory," The Journal of High Energy Physics, vol. 1998, no. 2, article 003, 1998.

[10] M. R. Douglas and C. M. Hull, "D-branes and the noncommutative torus," The Journal of High Energy Physics, vol. 1998, no. 2, article 008, 1998.

[11] F. Ardalan, H. Arfaei, and M. M. Sheikh-Jabbari, "Noncommutative geometry from strings and branes," The Journal of High Energy Physics, vol. 1999, no. 2, article 016, 1999.

[12] N. Seiberg and E. Witten, "String theory and noncommutative geometry," The Journal of High Energy Physics, vol. 1999, no. 9, article 032, 1999.

[13] C.-S. Chu and P.-M. Ho, "Non-commutative open string and Dbrane," Nuclear Physics B, vol. 550, no. 1-2, pp. 151-168, 1999.

[14] C.-S. Chu and P.-M. Ho, "Constrained quantization of open string in background $B$ field and non-commutative D-brane," Nuclear Physics B, vol. 568, no. 1-2, pp. 447-456, 2000.

[15] F. Ardalan, H. Arfaei, and M. M. Sheikh-Jabbari, "Dirac quantization of open strings and noncommutativity in branes," Nuclear Physics B, vol. 576, no. 1-3, pp. 578-596, 2000.

[16] Z. F. Ezawa, Quantum Hall Effects: Field Theoretical Approach and Related Topics, World Scientific, Singapore, 2000.

[17] G. Guo, Z. Yang, C. Long, and S. Qin, "DKP oscillator in noncommutative phase space," Canadian Journal of Physics, vol. 87, no. 9, pp. 989-993, 2009.

[18] Z.-H. Yang, C.-Y. Long, S.-J. Qin, and Z.-W. Long, “DKP oscillator with spin-0 in three-dimensional noncommutative phase space," International Journal of Theoretical Physics, vol. 49, no. 3, pp. 644-651, 2010.

[19] G. R. de Melo, M. de Montigny, and E. S. Santos, "Spinless Duffin-Kemmer-Petiau oscillator in a Galilean noncommutative phase space," Journal of Physics: Conference Series, vol. 343, article 012028, 2012.

[20] M. Falek and M. Merad, "DKP oscillator in a noncommutative space," Communications in Theoretical Physics, vol. 50, no. 3, pp. 587-592, 2008.

[21] M. H. Pacheco, R. R. Landim, and C. A. S. Almeida, "Onedimensional Dirac oscillator in a thermal bath," Physics Letters $A$, vol. 311, no. 2-3, pp. 93-96, 2003.

[22] A. Boumali and H. Hassanabadi, "The thermal properties of a two-dimensional Dirac oscillator under an external magnetic field," The European Physical Journal Plus, vol. 128, p. 124, 2013.

[23] S. Minwalla, M. van Raamsdonk, and N. Seiberg, "Noncommutative perturbative dynamics," The Journal of High Energy Physics, vol. 2000, no. 2, article 020, 2000.

[24] R. J. Duffin, "On the characteristic matrices of covariant systems," Physical Review, vol. 54, no. 12, p. 1114, 1938.

[25] N. Kemmer, "The particle aspect of meson theory," Proceedings of the Royal Society A, vol. 173, pp. 91-116, 1939.

[26] G. Petiau, Académie Royalede Belgique. Classe des Sciences. Mémoires. Collection, vol. 16, no. 2, 1936.

[27] T. R. Cardoso, L. B. Castro, and A. S. de Castro, "Inconsistencies of a purported probability current in the Duffin-KemmerPetiau theory," Physics Letters A, vol. 372, no. 38, pp. 5964-5967, 2008. 
[28] R. K. Pathria, Statistical Mechanics, Pergamon Press, Oxford, UK, 1st edition, 1972.

[29] G. Arfken, Mathematical Methods for Physicists, Academic Press, Orlando, Fla, USA, 3rd edition, 1985.

[30] A. F. Nikiforov and V. B. Uvarov, Special Functions of Mathematical Physics, Birkhäuser, Basel, Switzerland, 1988.

[31] A. Arda, R. Sever, and C. Tezcan, "Approximate analytical solutions of the Klein-Gordon equation for the Hulthén potential with the position-dependent mass," Physica Scripta, vol. 79, no. 1, Article ID 015006, 2009. 

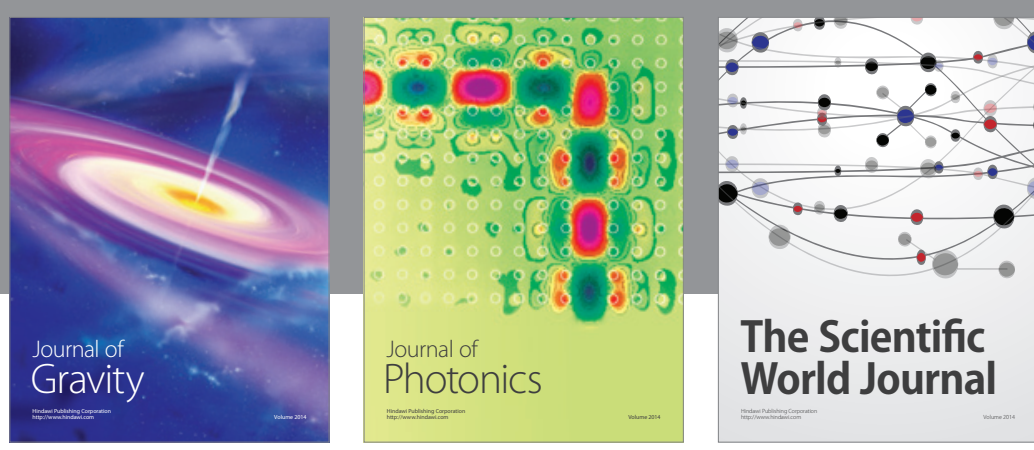

The Scientific World Journal
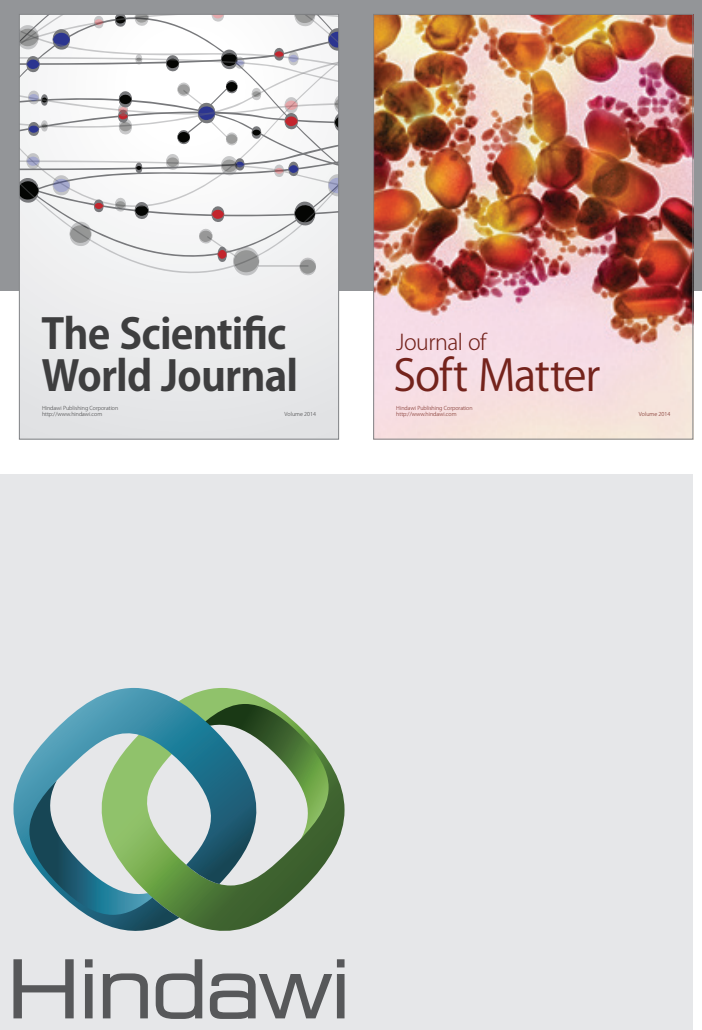

Submit your manuscripts at

http://www.hindawi.com

nternational Journal of

Statistical Mechanics
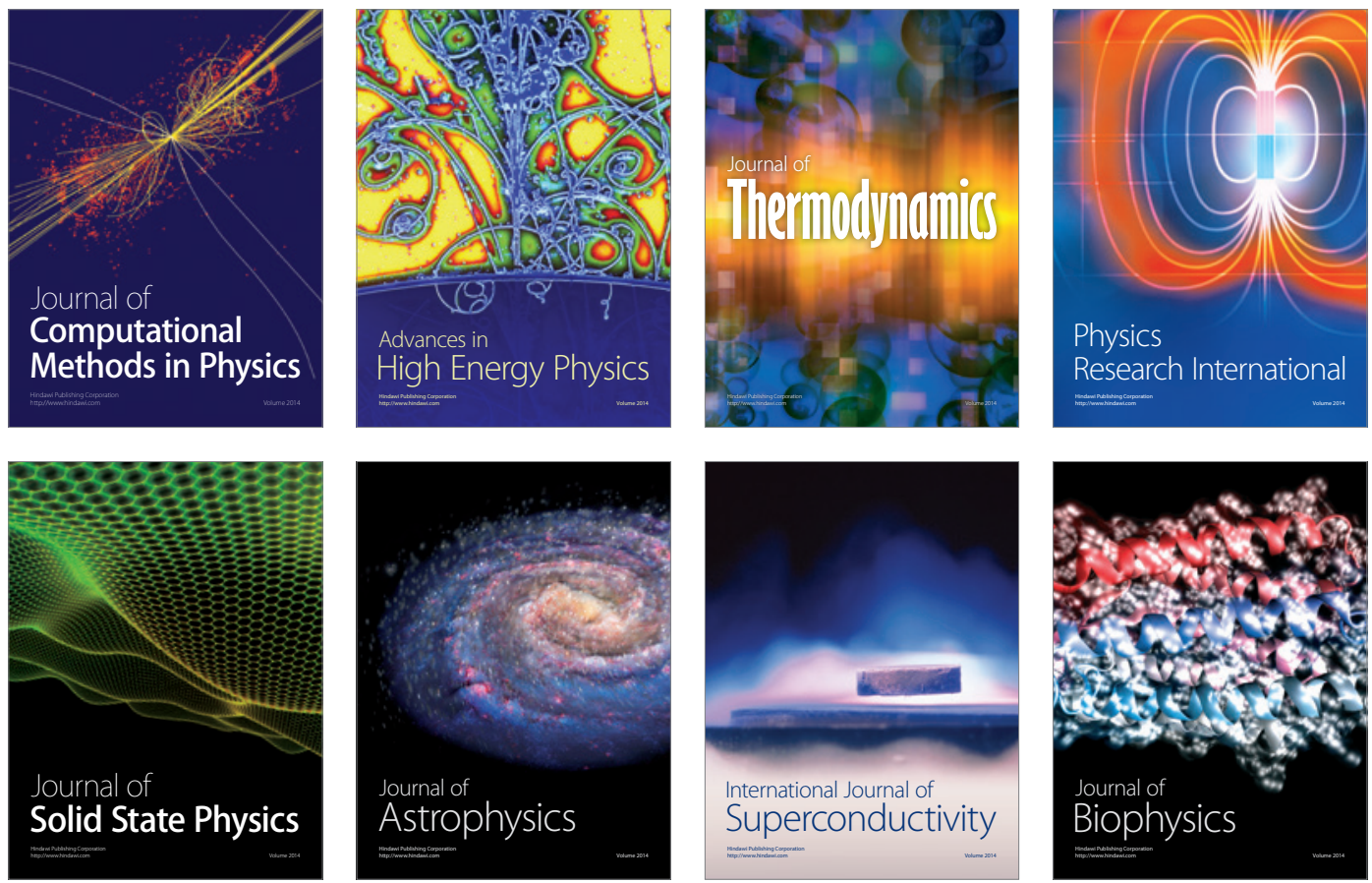
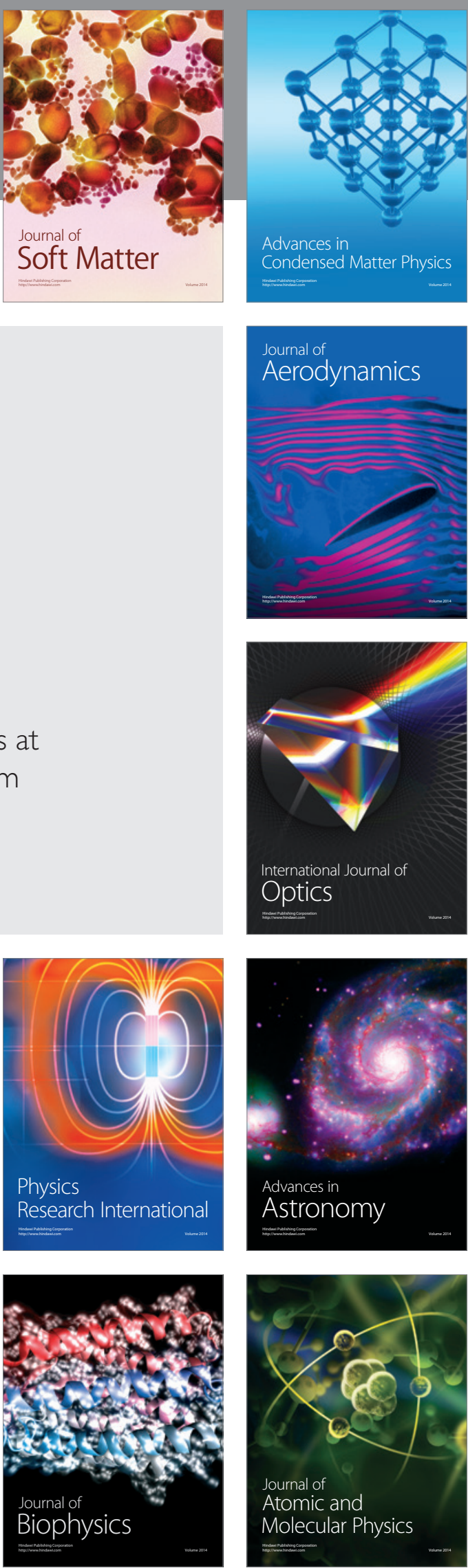